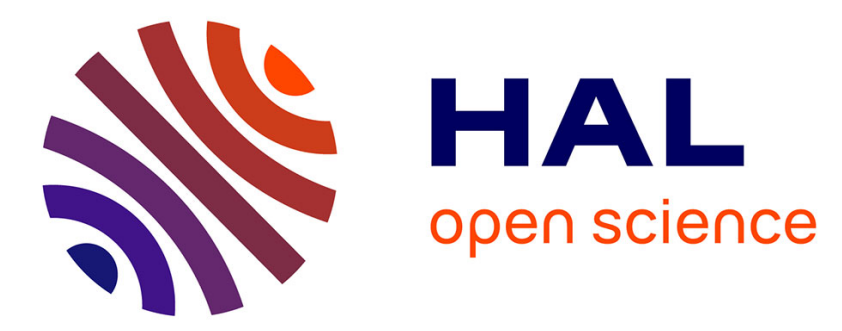

\title{
Reorientation Method to Suppress Simulator Sickness in Home VR Contents Using HMD
}

\author{
Yuki Ueda, Kazuma Nagata, Soh Masuko, Junichi Hoshino
}

\section{To cite this version:}

Yuki Ueda, Kazuma Nagata, Soh Masuko, Junichi Hoshino. Reorientation Method to Suppress Simulator Sickness in Home VR Contents Using HMD. 17th International Conference on Entertainment Computing (ICEC), Sep 2018, Poznan, Poland. pp.228-234, 10.1007/978-3-319-99426-0_22 . hal02128640

\section{HAL Id: hal-02128640 \\ https://hal.inria.fr/hal-02128640}

Submitted on 14 May 2019

HAL is a multi-disciplinary open access archive for the deposit and dissemination of scientific research documents, whether they are published or not. The documents may come from teaching and research institutions in France or abroad, or from public or private research centers.
L'archive ouverte pluridisciplinaire HAL, est destinée au dépôt et à la diffusion de documents scientifiques de niveau recherche, publiés ou non, émanant des établissements d'enseignement et de recherche français ou étrangers, des laboratoires publics ou privés. 


\title{
Reorientation Method to Suppress Simulator Sickness in Home VR Contents Using HMD
}

\author{
Yuki Ueda ${ }^{1^{*}}$, Kazuma Nagata ${ }^{1^{*}}$, Soh Masuko ${ }^{2}$, and Junichi Hoshino ${ }^{1}$ \\ ${ }^{1}$ University of Tsukuba, Graduate school of Systems and Information Engineering, \\ 1-1-1, Tennodai, Tsukuba-shi, Ibaraki, Japan \\ \{ueda,nagata\}@entcomp.esys.tsukuba.ac.jp,jhoshino@esys.tsukuba.ac.jp \\ ${ }^{2}$ Rakuten, Inc., Rakuten Institute of Technology, \\ Rakuten Crimson House, 1-14-1, Tamagawa, Setagaya-ku, Tokyo, Japan \\ so.masuko@rakuten.com
}

\begin{abstract}
While home-use HMD including Oculus Rift has been widely spread in the market today, simulator sickness mainly caused by difference between visual information and body sensation has taken up as a problem. Even though it has been proved that simulator sickness is reduced by reflecting actual physical movement to a VR space, many of approaches ever proposed had various restrictions and mechanisms easy to cause simulator sickness. In the current study, such an approach for moving within a VR space is proposed for home-use HMD that is less likely to cause simulator sickness.
\end{abstract}

Keywords: virtual reality, walking, redirection, simulator sickness

\section{Introduction}

With the spread of the home-use HMD today, various VR contents will be experienced at home. The applications of VR games, which gives high sense of immersion, to the open world games are expected. The latter enjoys growing popularity as the user can freely explore within a big stage. Also, if VR is utilized in online shopping, it would be possible to create the experiences that give the user the feeling of actually being in a shopping mall while at home, enjoying shopping within the VR showroom in which various information such as the user's preferences and the purchasing purpose, as well as the best-selling items and limited-time sales are reflected.

However, the foregoing experiences may cause the VR sickness accompanied by the symptoms such as fatigue, headache, eye strain, dizziness and nausea, because the user are made to be wandering about within the VR space for a long time. The discomfort with the VR sickness that makes long-hour VR experience painful for the user has also become an unavoidable obstacle for the developers delivering the content. While the cause of the VR sickness is still under discussion, one of the current leading theory is that it is mainly caused by the gap in somatic sensation between the real world and the VR space [1]. Although a lot of methods with which the user can move within the VR space by actually moving his body have been proposed so far aiming to enhance the immersive sensation or suppress the 
aforementioned gap in the somatic sensation, many of them have not been suitable for the VR experience at home as they require a big space and large-scale equipment. When moving within VR space using the position estimation technology with the help of a redirection method such as Redirected Walking [2] and Reorientation Technique [3], you can actually travel within the VR space by walking without being required to prepare a big experience space and large-scale equipment. However, the mechanism of correcting the direction when arriving at the end of the walkable area might cause the gap in somatic sensation and induce the VR sickness.

Therefore, in this study, we are proposing the redirection method that may be used in VR experiences at home, which enables the correction of direction without causing the gap in somatic sensation to realize the traveling method within the VR space which suppresses the VR sickness.

\section{Related Work}

The studies on the trend and countermeasures for the VR sickness that occurs when using the military simulator have been conducted based on the data obtained from many subjects. Kennedy et al. has developed the Simulator Sickness Questionnaire (SSQ) that is a subjective evaluation method using questionnaires [4]. The SSQ is designed to make the subjects evaluate themselves for the items about 16 body abnormalities using four grades, which enables measurement of VR sickness level with 4 types of indices; SSQ-TS (Total Score), SSQ-N (Nausea), SSQ-O (Oculomotor) and SSQ-D (Disorientation). The SSQ is still used in many studies as an effective indicator to measure the level of VR sickness with various symptoms.

LaVopla Jr. examined the mechanism causing VR sickness from a medical point of view and discussed three hypotheses [1]. Among them, the sensory conflict theory that is a hypothesis that the VR sickness is caused by the difference in somatic sensation is said to be the most widely accepted hypothesis.

Prothero et al. demonstrated the fact that It is possible to alleviate the VR sickness by making the user perceive as if the foreground environment is moving around him rather than he is traveling within the VR space by placing the Independent Visual Background [5] that can be glanced over from him, within the VR space when he is viewing an immersive image in a static state.

Llorach et al. compared the level of VR sickness with SSQ, between the cases where a game controller is used and where the user is traveling within VR space by actually walking with HMD leveraging a location estimation technology. The result showed that the sickness was alleviated when the user was actually walking leveraging a location estimation technology. However, the real-world situations in which this method could be used were limited because as big space as the one to be used within the VR space must be prepared [6].

Razzaque et al. proposed Redirected Walking (RDW) that is a traveling method within VR space, which dan deliver the user the sense of keep walking by going back and forth within the limited experience space [2]. With RDW, the user must pass through the checkpoints set within the VR space while walking. This technique 
enables the user to move within a bigger VR space than the experience space by making a gap in the rotation angle between the VR space and the real world when the user is correcting the direction at checkpoints. However, the user cannot travel freely because it requires settings of checkpoints within the VR space, thus the walking path is limited by it. Also, in comparison to the traveling method using a controller, the same lever of VR sickness had been observed with this technique in the SSC score. This may be due to the gap in the rotation angle generated when correcting the direction.

\section{System Overview}

In this study, we have defined the following four requirements for the traveling method that suppresses the VR sickness caused by home-use VR contents and considered the basic methods to be used.

1. Traveling within the VR space is enabled by actually walking.

2. The big experience space is not required.

3. The traveling path is not limited.

4. The gap in somatic sensation is not created.

To meet the foregoing requirements, we decided to take the approach that gives the user the sense of continuously walking within a big VR space by walking back and forth within the small walkable area. In the traditional studies on such as Redirected Walking [2] and Reorientation Technique [3], the traveling path is limited and the rotation angle that is different from the actual angle is visually delivered not to make the user perceive they are correcting the direction. On the other hand, in this study we have tried to alleviate the VR sickness by intentionally make the user recognize he is correcting the direction and let him perceive the proper rotation angle.

For this purpose, first we decided to rotate the foreground, a view seen from the user within the VR space when he is correcting the direction, in line with the rotation by the user so that he can keep traveling after correcting the direction. However, only rotating the foreground in line with the user's rotation will end up making the user see the static scenery despite the fact that he is rotating in the real world. Thus, in this study, based on our insight to the independent visual landscape [5], we have positioned the visual background fixed to the world coordinate system within the VR space to be seen by the user as shown in Figure 1. With this, the sensation as if the foreground is rotating in line with the user's body is generated when he is correcting the direction, and the redirection method that does not cause the gap in somatic sensation due to the rotation angle may be realized. Based on this idea, we have implemented a system.

The system is consisted of a PC to draw VR environment, HTC vive; a home-use HMD that has a position estimation function, a base station for HTC vive and a flat floor. The user is freely transitioning between the following two phases by manipulating the controller for HTC vive.

1) Traveling phase In the traveling phase, the user can freely walk within the walkable area. While the user is walking, the position and orientation of the HMD is tracked, and directly reflected in his view within the VR space. When the user arrives 
at the edge of the walkable area, the grid indicating the walkable area is shown on the HMD, indicating it is impossible to go further ahead. To go further ahead within the VR space, the user must transition to the rotation phase by manipulating the controller to correct the direction.

2) Rotation phase The user corrects the direction under the state where the foreground and the user's position are fixed. This has been enabled by rotating the foreground around the HMD position coordinate at the center, by the range of the rotation around the yaw axis of the HMD. The visual background fixed to the world coordinate system is shown to the user to provide the visual information without any gap in rotation angle. This delivers the sensation to the user as if the foreground is rotating with his body when correcting the direction. This time, as in Figure 2, the visual background was made to be always seen from the user during the rotation phase by rendering the Sphere positioned to cover the whole foreground like a wire frame. The Sphere was fixed to the world coordinate system independent of the user's movement and rotation. During the rotation phase, an arrow that indicates the user's rotation direction is shown on the controller. On completion of the direction correction, the user is transitioned to the traveling phase after being notified as in Figure 3 .

visual background fixed to the world coordinate system

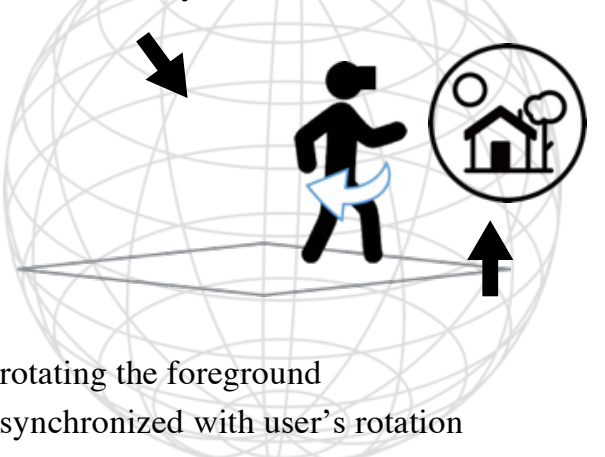

Fig.1 Fixed visual background

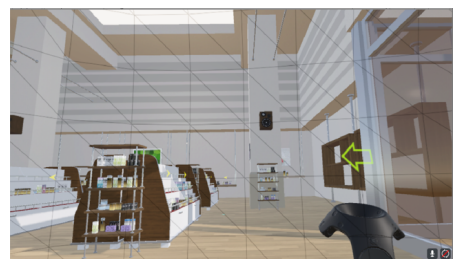

Fig.2 Visual background from user's view

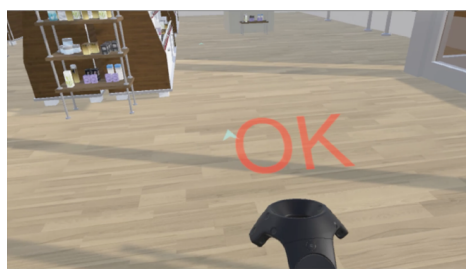

Fig.3 Completion of the direction correction

\section{Experiments}

This time, in place of the rotation phase for the proposed system, we have prepared a model created based on the conventional method, which involves the redirection method that enables correction of direction by creating a gap in the rotation angle to compare the proposed method and the conventional redirection method

For the evaluation experiment, we have used the PC built-in with NVIDIA GeForce GTX 970 4GB as CPU to generate the VR space. As shown in Figure 4, while experiencing the system, the subject was holding the controller to transition between the phases with his right hand and walking within the flat area of $3.0 \mathrm{~m} \mathrm{x}$ 
$1.5 \mathrm{~m}$. The subject was accompanied by an assistant to watch the cable not to be entangled with his body during the experiment.

We had 10 male subjects in their 20's participated in the experiment. They are divided into two groups of 5 people. Both groups experienced the proposed system and the conventional method model alternately with 30 minutes break in between, so that everyone is experimented with both redirection methods. For this experiment, the scene mimicking the VR showroom as shown in Figure 5 below had been prepared. These subjects were given the task to walk through the path that had been explained beforehand to reach the goal point. The arrows to indicate the direction to walk toward were shown on the floors and shelves in the scene. The pathway walked through by the subjects is shown in Figure 6. The total walking distance is about $50 \mathrm{~m}$. The two redirection methods were compared in terms of the time to reach the goal, the time to correct the direction and the number of direction corrections, the SSQ [4] evaluation value right after the experiment and the contents of the hearing from the subjects.

The time to finish the task, the time to correct directions, the average number of direction corrections and the standard deviations for the subjects are shown in Table 1. With this experiment, it has been found that for the proposed system, the time to correct directions is $34 \%$ shorter and the time to complete the task is $21 \%$ shorter than the conventional method model. It is obvious that the difference of the time required to complete the task between two methods corresponds to the difference of the time taken to correct directions. As a result of the t-test that supports each element, significant differences have been shown for all of the time to complete $(\mathrm{t}(9)=2.716$, $\mathrm{p}<.05)$, the time to correct directions $(\mathrm{t}(9)=3.647, \mathrm{p}<.01)$ as well as the number of direction corrections $(\mathrm{t}(9)=2.358, \mathrm{p}<.05)$,

Also, the evaluation values on SSQ-TS (Total Score), SSQ-N(Nausea), SSQ-O (Oculomotor) and SSQ-D (Disorientation) are shown in Table 2 below. Furthermore, as a result of the non-parametric Wilcoxon signed-rank test for each evaluation value, the proposed system has shown a significantly low value on SSQ-D $(T=1, \mathrm{p}<.05)$ compared to the conventional method model. Although significant differences were not shown on other scores, there was a dominant trend for the better on the subjects participated in this experiment.

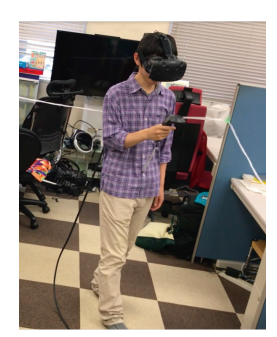

Fig.4 Scene of experiments

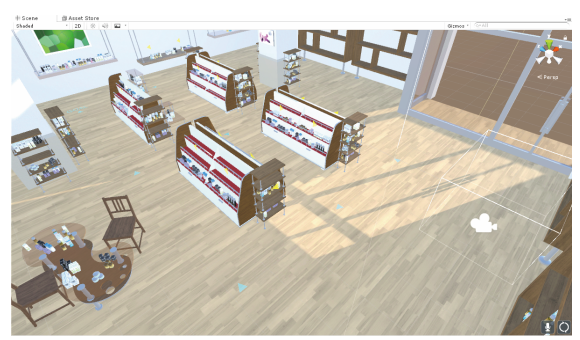

Fig.5 VR showroom setup

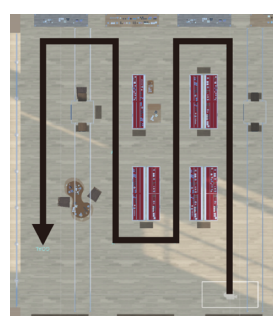

Fig.6 pathway walked through by the subjects 
Table 1. Comparison of times

\begin{tabular}{|c|cc|cc|}
\hline & \multicolumn{2}{|c|}{$\begin{array}{c}\text { Conventional } \\
\text { method }\end{array}$} & \multicolumn{2}{c|}{ Proposed method } \\
\cline { 2 - 5 } & Average & SD & Average & SD \\
\hline $\begin{array}{c}\text { Time to finish } \\
\text { tasks * }\end{array}$ & $298 \mathrm{sec}$. & $58 \mathrm{sec}$. & $236 \mathrm{sec}$. & $37 \mathrm{sec}$. \\
\hline $\begin{array}{c}\text { Time to correct } \\
\text { directions** }\end{array}$ & $131 \mathrm{sec}$. & $37 \mathrm{sec}$. & $87 \mathrm{sec}$. & $17 \mathrm{sec}$. \\
\hline $\begin{array}{c}\text { Average } \\
\text { number of } \\
\text { corrections* }\end{array}$ & $31 \mathrm{times}$ & $4.1 \mathrm{times}$ & 27 times & 3.5 times \\
\hline
\end{tabular}

$(* \cdots p<.05, \quad * * \cdots p<.01)$

Table 2 Comparison of SSQ score

\begin{tabular}{|c|cc|cc|}
\hline & \multicolumn{2}{|c|}{$\begin{array}{c}\text { Conventional } \\
\text { method }\end{array}$} & \multicolumn{2}{c|}{$\begin{array}{c}\text { Proposed } \\
\text { method }\end{array}$} \\
\cline { 2 - 5 } & Average & SD & $\begin{array}{c}\text { Avera } \\
\text { ge }\end{array}$ & SD \\
\hline SSQ- & 48.25 & 47.35 & 23. & 19.78 \\
TS & 33.39 & 40.31 & $\begin{array}{c}14 . \\
31\end{array}$ & 14.93 \\
SSQ-N & 35.63 & 34.08 & $\begin{array}{c}20 . \\
47\end{array}$ & 16.97 \\
SSQ-0 & 35.67. & 31.74 \\
SSQ-D* & 65.42 & 59.07 & 84 & $\left({ }^{*} \cdots \mathrm{p}<.05\right)$ \\
\hline
\end{tabular}

The time to correct directions is shorter for the proposed system compared to the conventional method model. This is because while the conventional method model recommends the rotation direction with bigger rotation angle to minimize the gap in somatic sensation upon correction of direction, the proposed system recommends the direction that can complete correction of direction within the minimum time. The shorter time to correct directions helps suppressing sickness from the rotation. It also helps suppressing VR sickness due to the shortened total experience time.

The reason why a significant difference was shown in the number of direction corrections would be because the user lost sight of the planned path before the direction change and unable to systematically select the path as seen in the many opinions, which says, "turning around within the VR space made me forget which way I wanted to go after correcting the direction."

From the result of SSQ, it has been turned out that the proposed system had a significantly better result compared to the conventional method model as for the disorientation after the experience (indicated by SSQ-D). Based on this result as well as the fact found through the hearing that the majority of them had perceived the difference in rotation angle with the conventional method model and most of them 
had felt uncomfortable, we think that the proposed system could suppress the VR sickness from disorientation better than the traditional method. Also, the shorter time to correct directions in the proposed system compared to the conventional method may have been contributed to suppress the VR sickness. However, if the rotation direction with narrow rotation angle is recommended to shorten the time to correct directions in the conventional method model, the gap in somatic sensation would become very big, leading to the bigger difference in the disorientation score. Also, for other scores, while no significant differences were shown from the test, the proposed system showed lower scores for each SSQ index compared to the conventional method model. Thus, we think that the level of VR sickness was suppressed with the proposed system. Although there seems to be some differences in the average SSQ scores between these systems at a glance, no significant difference has been shown on the scores except for SSQ-D. This result may be caused by the short experience time and the light task, which ended up with light level of sickness for both methods. For this reason, we think significant difference may be observed between both systems in other evaluation values as well by imposing heavier task

\section{Conclusion}

In this study, the redirection method that make the users perceive the correct rotation angle by showing them a static background upon change of direction had been proposed and its effectiveness has been revealed with the experiment. With this experiment, it is shown that the traveling method that can suppress VR sickness is enabled within the experience space prepared at home. However, the user must stop the main VR experience every time when changing the direction for both methods. Therefore, in terms of timeliness, these methods may be inferior to other traveling method. As such, these methods would be preferable to be used in the games with less action element, or for the shopping experiences in the VR showroom dealing with the items that is not for sale or limited.

\section{References}

[1] LaViola Jr, J. 2000. A discussion of cybersickness in virtual environments. ACM SIGCHI Bulletin, 32(1):47-56.

[2] Razzaque, S., Kohn, Z., Whitton, M. 2001. Redirected Walking. EuroGraphics 2001.

[3] Peck, T., Whitton, M., Fuchs, H. 2008. Evaluation of Reorientation Techniques for Walking in Large Virtual Environments. Virtual Reality Conference, 2008. VR '08. IEEE. 
[4] Kennedy R. S., Lane, N. E., Lilienthal, M. G. 1993. Simulator sickness questionnaire: An enhanced method for quantifying simulator sickness. The International Journal of Aviation Psychology, 3(3):203-220.

[5] Prothero, J.D., Draper, M.H., Furness, T.A., Parker, D.E., Wells, M.J. 1999. The use of an independent visual background to reduce simulator side-effects. Aviation, Space, and Environmental Medicine, 70(3):135-187.

[6] Llorach, G., Evans, A., Blat, J. 2014. Simulator Sickness and Presence using HMDs: comparing use of a game controller and a position estimation system. VRST '14 Proceedings of the 20th ACM Symposium on Virtual Reality Software and Technology, 137-140. 